\title{
Editorial
}

\section{Stochastic Systems and Control: Theory and Applications}

\author{
Weihai Zhang, ${ }^{1}$ Honglei $\mathrm{Xu},{ }^{2}$ Huanqing Wang, ${ }^{3}$ and Zhongwei Lin ${ }^{4}$ \\ ${ }^{1}$ College of Electrical Engineering and Automation, Shandong University of Science and Technology, Qingdao 266590, China \\ ${ }^{2}$ Department of Mathematics and Statistics, Curtin University, Perth, WA, Australia \\ ${ }^{3}$ Department of Mathematics, Bohai University, Jinzhou, Liaoning 121000, China \\ ${ }^{4}$ State Key Laboratory of Alternate Electrical Power System with Renewable Energy Sources, North China Electric Power University, \\ Beijing 102206, China \\ Correspondence should be addressed to Weihai Zhang; w_hzhang@163.com
}

Received 20 November 2017; Accepted 21 November 2017; Published 27 December 2017

Copyright (C) 2017 Weihai Zhang et al. This is an open access article distributed under the Creative Commons Attribution License, which permits unrestricted use, distribution, and reproduction in any medium, provided the original work is properly cited.

The objective of this special issue is to address recent research trends and developments in stochastic control systems and their applications to control, filtering, communication, manufacturing, fault detection, and systems biology. A substantial number of papers were submitted, and after a thorough peer review process, some related papers were selected to be included in this special issue. These papers collected in this special issue highlight the contemporary topics in research related to stochastic systems control theory and applications and will introduce readers to the latest advances in the field.

The paper by X. Li et al. presents a decentralized coordinated control of double fed induction generators based on the neural interaction measurement observer. The proposed controller can be formulated as a mixed optimization problem with constraints of PI structure and regional pole placement. Simulation results are presented to demonstrate the capabilities of the proposed control strategy.

The paper by X. Geng et al. considers A-diagnosability in distributed stochastic discrete event systems. A local model and global model are defined, and a synchronized stochastic diagnoser is constructed. The authors also propose a necessary and sufficient condition for a distributed SDES to be A-diagnosable.

The paper by J. Zhang et al. investigates a new stochastic chemostat model with two substitutable nutrients and one microorganism. Firstly, for the deterministic model, the threshold for extinction or existence of the microorganism is obtained by analyzing the stability of the equilibrium. Then the threshold of the stochastic chemostat for the extinction and the permanence in mean of the microorganism is explored.

The paper by $\mathrm{H}$. Huang analyzes one kind of linear quadratic stochastic control problem of forward backward stochastic control system associated with Lévy process. We obtain the explicit form of the optimal control, then prove it to be unique, and get the linear feedback regulator by introducing one kind of generalized Riccati equation. Finally, we discuss the solvability of the generalized Riccati equation, and its existence and uniqueness of the solutions are proved in a special case.

The paper by Y. Zhao and Y. Fu investigates the problems of stability and stabilization for stochastic Markovian jump systems subject to partially unknown transition probabilities and multiplicative noise. By introducing the free-weighing matrix method, the results obtained in this paper not only are less conservative than the existing ones but also can be regarded as extensions of the corresponding results of Markovian jump systems without noise.

The paper by P. Shang and L. Kong discusses the mixed matrix regression model to deal with the complex matrix and vector data. Under some mild conditions, it is proved that the degrees of freedom of mixed matrix regression model are the sum of the degrees of freedom of Lasso and regularized matrix regression.

The paper by G. Zhu et al. presents a neural network prespecified performance control scheme for a class of generic hypersonic flight vehicles with uncertain dynamics and stochastic disturbance. The main feature of the proposed 
scheme is that only one parameter needs to be estimated at each design step, so that the computational burden can be greatly reduced and the designed controller is much simpler.

The paper by M. Elloumi and S. Kamoun proposes an improved structural estimation procedure for large-scale interconnected nonlinear systems which are composed of a set of interconnected SISO Hammerstein structures and described by discrete-time stochastic models with unknown time-varying parameters. An extensive Determinant Report algorithm is developed to determine the order of the process. An improved discrete-time technique based on Recursive Extended Least Squares with Varying Time Delay method is proposed to estimate the time delays of the considered system. The developed theoretical analysis and simulation results prove the validity and performance of the proposed algorithms.

The paper by T. Hou et al. deals with the mixed control problem through the solvability of four coupled difference matrix-valued recursions for discrete-time infinite Markov jump systems. And a numerical example is given to illustrate its efficiency.

The paper by R. Caballero-Águila et al. addresses the least squares centralized fusion estimation problem of discretetime random signals from measured outputs. By an innovation approach and using the last observation that successfully arrived when a packet is lost, a recursive algorithm is designed for the filtering estimation problem. The proposed algorithm is easily implemented and does not require knowledge of the signal evolution model, as only the first- and second-order moments of the processes involved are used.

The paper by Z. Li et al. presents a novel fifth-degree cubature Kalman filter to improve the accuracy of real-time orbit determination by ground-based radar. The simulation results show that, compared with the traditional third-degree algorithm, the proposed fifth-degree algorithm has a higher accuracy of orbit determination.

The paper by $\mathrm{C}$. Zhou et al. investigates a two-stage stochastic quadratic programming problem with inequality constraints. By quasi-Monte-Carlo-based approximations of the objective function, a feasible sequential system of linear equations method is proposed. The convergence rate is locally superlinear under some additional conditions.

This paper by W. Sun et al. investigates the control problem of magnetic levitation system, in which velocity feedback signal is influenced by stochastic disturbance. Single-degree-freedom magnetic levitation is regarded as an energy-transform action device. Based on the Hamiltonian structure, the control law of magnetic levitation system is designed by applying Lyapunov theory.

The paper by Z. Zheng et al. investigates preferential random walks (PRW) on weighted complex networks. By using two different analytical methods, two exact expressions are derived for the mean first passage time between two nodes. This work may provide a comprehensive approach for exploring random walks on complex networks, especially biased random walks, which could help to better understand and tackle some practical problems such as search and routing on networks.
The paper by L. Zhang et al. presents multienergy hybrid system structure and constructs a scheduling model with the objective functions of maximum economic benefit and minimum power output fluctuation. Robust stochastic theory is introduced to describe uncertainty and propose a multiobjective stochastic scheduling optimization mode by transforming constraint conditions with uncertain variables.

The paper by P. Wang et al. presents an efficient approximation of the Labeled Multi-Bernoulli Filter to perform online multitarget state estimation and track maintenance efficiently. Then, a target posterior approximation technique is proposed to use a weighted single Gaussian component representing each individual target. Numerical results verify that the proposed efficient approximation of the LMB filer achieves accurate tracking performance.

The paper by M. Liu et al. investigates a stochastic optimal control problem where the control system is driven by ItôLévy process. The necessary condition about existence of optimal control for stochastic system by using traditional variational technique under the assumption that control domain is convex is proved. As an application to finance, an example of recursive consumption utility optimization problem is used to illustrate the practicability of our result.

The paper by $\mathrm{H}$. Hu et al. considers a stock price modeled by a geometric-Brownian motion which features volatility uncertainty to analyze the financial markets with volatility uncertainty. A new arbitrage-free concept is utilized to obtain the detailed results which give us an economically better understanding of financial markets under volatility uncertainty.

The paper by P. S. Kim proposes an alternative finite memory structure (FMS) smoother with a recursive form under a least squares criterion using a forgetting factor strategy. The proposed FMS smoother is shown to have good inherent properties such as time-invariance, unbiasedness, and deadbeat. It is shown that the proposed FMS smoother can be better than the existing FMS smoother for incorrect noise covariance and the IMS smoother for temporary uncertainties.

The paper by N. Yuguang et al. presents a novel method combining Markov chain model and generalized projection nonnegative matrix factorization to detect and diagnose the faults in industrial process. The proposed method is applied to a $1000 \mathrm{MW}$ unit boiler process. The simulation results clearly illustrate the feasibility of the proposed method.

The paper by J. Kong et al. proposes a novel deep neural network model for prune reordering table. The reordering model in PBMT by filtering reordering table is optimized using a recursive autoencoder model. The experimental results show that the proposed approach obtains high improvement in BLEU score with lower scale of reordering table on two language pairs: English-Chinese and UyghurChinese MT.

The paper by X. Cai et al. proposes a novel method on selection of an initial covariance matrix and a horizon for the Kalman filter to make sure that a sequence of the closed-loop Kalman filters are stable as time-invariant filters at subsequent time instant. The convergent properties and the stability conditions are less conservative since they provide 
analytic results and are applicable to more common cases where the RDEs are not monotonic.

The paper by M. Cui et al. considers the model and control problem of nonlinear active suspension in rough road. By an appropriate transform, the model is transformed into a lower triangular system, which can be used as backstepping method. Then a controller is designed such that the mean square of the state converges to an arbitrarily small neighborhood of zero by tuning design parameters. The simulation results illustrate the effectiveness of the proposed scheme.

The paper by X. Leng et al. proposes a stochastic SIVS epidemic system with nonlinear saturated infection rate under vaccination and investigates the dynamics predicted by the model. By using Itô's formula and Lyapunov methods, this paper investigates the existence and uniqueness of global positive solution. It is shown that large stochastic noises can lead to the extinction of epidemic diseases. Finally, the numerical simulations demonstrate the performance of the theoretical findings.

The paper by K. Nakade and $H$. Niwa discusses the effect by the lead time quotation in an $M / M / 1$ base stock manufacturing-inventory queue into the customer's utility function. The numerical examples show that the optimal lead time quotation policy for maximizing system's average profit has low customer's utility, and the other simple heuristic quotation policy leads to the greater expected values of customers' utility.

The paper by S. Li et al. constructs a new class of interest models considering stochastic behavior of interest rates in financial market by compound Poisson process. Simulations are used to illustrate the theoretical results and the effect of parameters in interest model on actuarial present values is also analyzed.

The paper by N. Duan et al. considers a more general stochastic nonlinear time delay system driven by unknown covariance noise and investigates its adaptive state-feedback control problem. By Lyapunov-Krasovskii functionals and adaptive backstepping, an adaptive state-feedback controller is constructed by overcoming the negative effects brought by unknown time delay and covariance noise. A simulation example demonstrates the effectiveness of the proposed scheme.

The paper by J. Ma et al. considers an optimal liquidation by using the Almgren-Chriss market impact model on the background that the agents liquidate assets completely. The stochastic component of the price process is eliminated from the mean-variance. The Nash equilibrium is considered as the second-order linear differential equation with variable coefficients. The existence and uniqueness of solutions are proved for the differential equation with two boundaries. The numerical examples and properties of the solution are given.

The paper by Y. Yi et al. studies a cooperative stochastic differential game of transboundary industrial pollution between two asymmetric nations in infinite-horizon level. The feedback Nash equilibrium strategies of governments and industrial firms are discussed. It is found that the governments being cooperative in transboundary pollution control will set a higher pollution tax rate and make more pollution abatement effort than when they are noncooperative.
The paper by L. Li et al. discusses stochastic multi-item capacitated lot-sizing problems with and without setup carryovers, S-MICLSP and S-MICLSP-L. A new fix-and-optimize approach is developed to solve the approximated models. Numerical experiments are performed on the instances following the nature of realistic steel products.

The paper by S. Kim et al. considers a multiperiod inventory control model in which a risk averse firm faces loss averse customer's uncertain demand and makes an inventory replenishment and pricing decision by maximizing the firm's expected utility.

The paper by M. Yu et al. considers the global exponential antisynchronization in mean square of memristive neural networks with stochastic perturbation and mixed time-varying delays. Two kinds of novel delay-dependent and delay-independent adaptive controllers are designed. On the basis of the differential inclusions theory, inequality theory, and stochastic analysis techniques, several sufficient conditions are obtained to guarantee the exponential antisynchronization between the drive system and response system.

The paper by X. Li et al. presents a novel multivariate Bayesian control approach which enables the implementation of early fault detection for a helicopter gearbox with cost minimization maintenance policy under varying load. The effective computational algorithm in the semi-Markov decision process framework is designed to obtain the optimal control limit. A comparison with the multivariate Bayesian control chart based on hidden Markov model and the traditional age-based replacement policy is given which illustrates the effectiveness of the proposed approach.

The paper by L. Wang et al. presents the state space and transition rate matrix corresponding to the 6-component star Markov repairable system with spatial dependence via probability analysis method. Several reliability indices, such as the availability, the probabilities of visiting the safety, the degradation, the alert, and the failed state sets, are obtained by Laplace transform method and a numerical example is provided to illustrate the results.

The paper by Q. Cao et al. discusses the low frequency random load of a tractor. An innovative method is presented for theoretical shift schedule modification by the fuzzy algorithm, based on the random load standard deviation and the alteration rate of both steady state values of the load and of the throttle position. The simulation results demonstrated that the modified shift schedule could discover the running state of the tractor.

The paper by S. Ji and X. Shi concerns the recursive utility maximization problem for terminal wealth under partial information. The considered problem under partial information is first transformed into the one under full information. Then, the ignorance case and explicit saddle points of several examples are obtained. At last, when the generator of the recursive utility is smooth, the terminal perturbation method is applied to characterize the optimal terminal wealth.

Although the selected topics and published papers may not represent all of the recent developments in the area, we hope that readers find the special issue helpful and useful. 


\section{Acknowledgments}

We sincerely thank all of the reviewers for their valuable comments and suggestions which have contributed greatly to the quality of the special issue.

Weihai Zhang Honglei $X u$

Huanqing Wang

Zhongwei Lin 


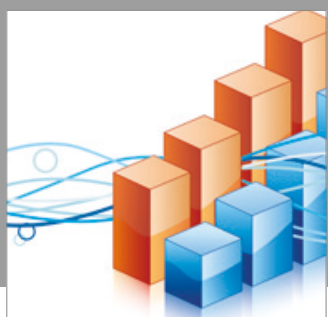

Advances in

Operations Research

vatersals

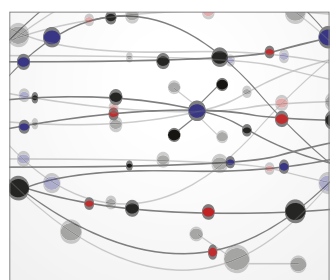

\section{The Scientific} World Journal
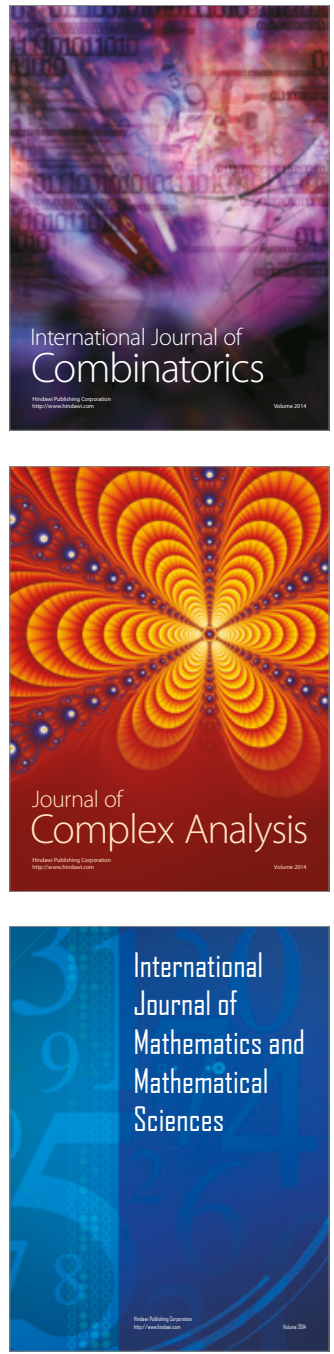
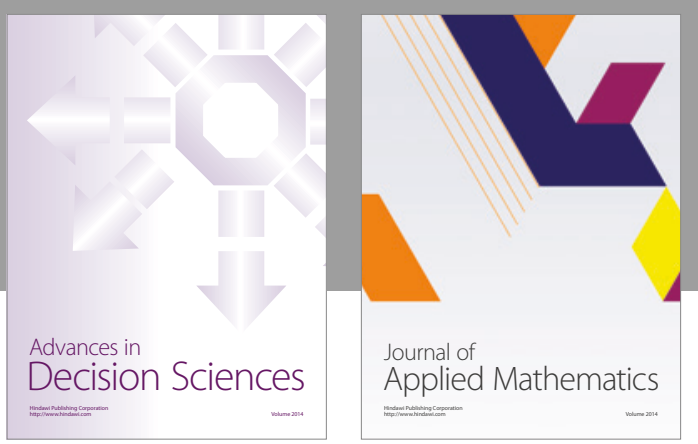

Algebra

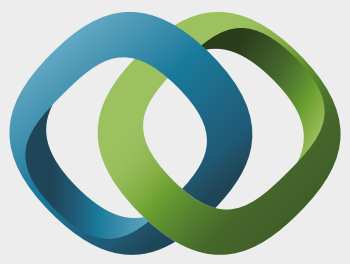

\section{Hindawi}

Submit your manuscripts at

https://www.hindawi.com
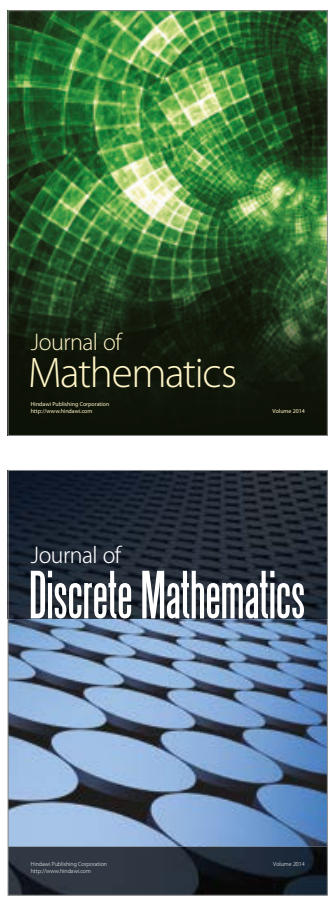

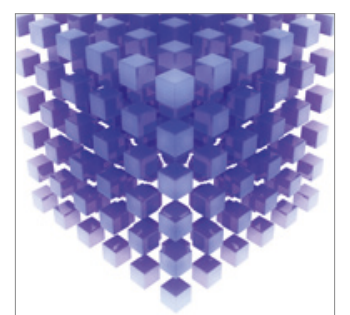

Mathematical Problems in Engineering
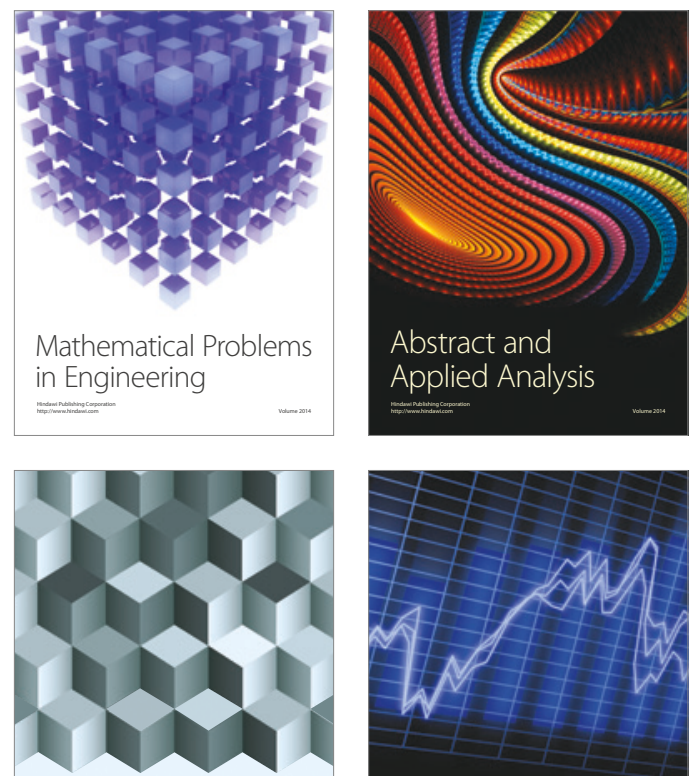

Journal of

Function Spaces

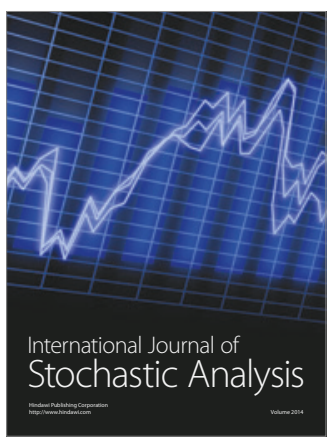

Probability and Statistics
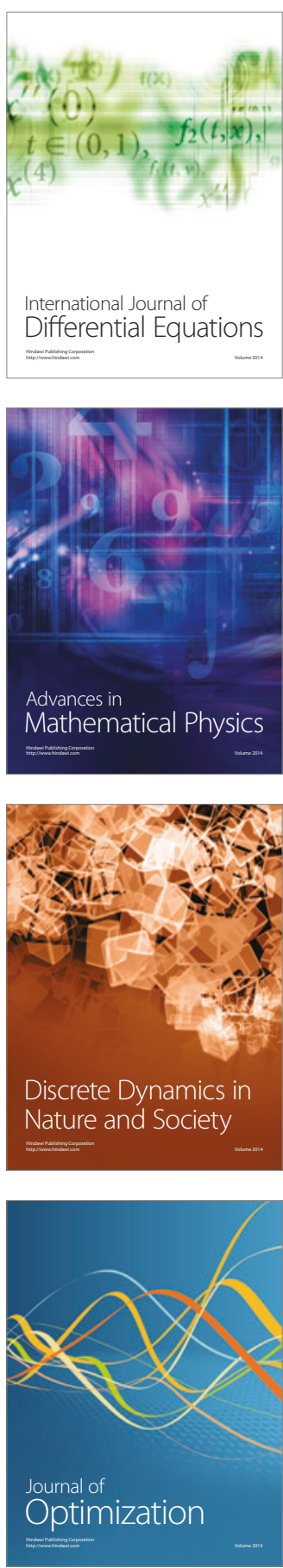